Appl. Set-Valued Anal. Optim. 4 (2022), No. 1, pp. 27-39

Available online at http://asvao.biemdas.com

https://doi.org/10.23952/asvao.4.2022.1.02

\title{
STRUCTURE OF OPTIMAL TRAJECTORIES OF DYNAMICAL SYSTEMS WITH A LYAPUNOV FUNCTION
}

\author{
ALEXANDER J. ZASLAVSKI
}

Department of Mathematics, The Technion - Israel Institute of Technology, 32000 Haifa, Israel

\begin{abstract}
In this paper we study the structure of optimal trajectories for discrete disperse dynamical systems generated by the set-valued mappings introduced by Rubinov in 1980 .

Keywords. Compact metric space; Dynamical system; Global Attractor; Optimal trajectories; Turnpike.
\end{abstract}

\section{INTRODUCTION}

In $[1,2]$, Rubinov introduced a discrete disperse dynamical system generated by a set-valued mapping acting on a compact metric space, which were recently studied in [1]-[7]. This disperse dynamical system has prototype in the mathematical economics $[1,8,9]$. In particular, it is an abstract extension of the classical von Neumann-Gale model [1, 8, 9]. Our dynamical system is described by a compact metric space of states and a transition operator which is set-valued. Dynamical systems theory has been a rapidly growing area of research which has various applications to physics, engineering, biology, and economics. In this theory, one of the goals is to study the asymptotic behavior of the trajectories of a dynamical system. Usually, in the dynamical systems theory, a transition operator is single-valued. In [1]-[7], we studied the dynamical systems with a set-valued transition operator. Such dynamical systems correspond to certain models of economic dynamics $[1,8,9]$.

Let $(X, \rho)$ be a compact metric space, and let $a: X \rightarrow 2^{X} \backslash\{\emptyset\}$ be a set-valued mapping whose graph

$$
\operatorname{graph}(a)=\{(x, y) \in X \times X: y \in a(x)\}
$$

is a closed subset of $X \times X$. For each nonempty subset $E \subset X$, set

$$
a(E)=\cup\{a(x): x \in E\} \text { and } a^{0}(E)=E .
$$

By induction, we define $a^{n}(E)$ for any natural number $n$ and any nonempty subset $E \subset X$ as follows: $a^{n}(E)=a\left(a^{n-1}(E)\right)$.

In this paper, we study the convergence and structure of trajectories of the dynamical system generated by the set-valued mapping $a$. Following $[1,2]$, this system is called a discrete dispersive dynamical system.

E-mail address: ajzasl@technion. ac.il.

Received May 13, 2021; Accepted June 5, 2021.

(C)2022 Applied Set-Valued Analysis and Optimization 
A sequence $\left\{x_{t}\right\}_{t=0}^{\infty} \subset X$ is called a trajectory of $a$ (or just a trajectory if the mapping $a$ is understood) if $x_{t+1} \in a\left(x_{t}\right)$ for all integers $t \geq 0$.

Let $T_{2}>T_{1}$ be integers. A sequence $\left\{x_{t}\right\}_{t=T_{1}}^{T_{2}} \subset X$ is called a trajectory of $a$ (or just a trajectory if the mapping $a$ is understood) if $x_{t+1} \in a\left(x_{t}\right)$ for all integers $t \in\left\{T_{1}, \ldots, T_{2}-1\right\}$.

Define

$\Omega(a)=\left\{z \in X:\right.$ for each $\varepsilon>0$ there is a trajectory $\left\{x_{t}\right\}_{t=0}^{\infty}$ such that $\left.\liminf _{t \rightarrow \infty} \rho\left(z, x_{t}\right) \leq \varepsilon\right\}$.

Clearly, $\Omega(a)$ is a nonempty closed subset of $(X, \rho)$. In the literature the set $\Omega(a)$ is called a global attractor of $a$. Note that, in [1,2], $\Omega(a)$ is called a turnpike set of $a$. This terminology is motivated by mathematical economics $[1,8,9]$.

For each $x \in X$ and each nonempty closed subset $E \subset X$, put $\rho(x, E)=\inf \{\rho(x, y): y \in E\}$. It is clear that, for each trajectory $\left\{x_{t}\right\}_{t=0}^{\infty}, \lim _{t \rightarrow \infty} \rho\left(x_{t}, \Omega(a)\right)=0$. It is not difficult to see that if, for a nonempty closed set $B \subset X, \lim _{t \rightarrow \infty} \rho\left(x_{t}, B\right)=0$ for each trajectory $\left\{x_{t}\right\}_{t=0}^{\infty}$, then $\Omega(a) \subset B$.

Let $\phi: X \rightarrow R^{1}$ be a continuous function such that $\phi(z) \geq 0$ for all $z \in X$, and

$$
\phi(y) \leq \phi(x) \text { for all } x \in X \text { and all } y \in a(x) .
$$

It is clear that $\phi$ is a Lyapunov function for the dynamical system generated by the mapping $a$. It should be mentioned that in mathematical economics usually $X$ is a subset of the finitedimensional Euclidean space, and $\phi$ is a linear functional on this space; see, e.g., $[1,8,9]$. Our goal is to study approximate solutions of the following problem

$$
\begin{gathered}
\phi\left(x_{T}\right) \rightarrow \max , \\
\left\{x_{t}\right\}_{t=0}^{T} \text { is a program satisfying } x_{0}=x,
\end{gathered}
$$

where $x \in X$ and a natural number $T$ are given.

The following theorem was obtained in [7].

Theorem 1.1. The following properties are equivalent:

(1) if a sequence $\left\{x_{t}\right\}_{t=-\infty}^{\infty} \subset X$ satisfies $x_{t+1} \in a\left(x_{t}\right)$ and $\phi\left(x_{t+1}\right)=\phi\left(x_{t}\right)$ for all integers $t$, then $\left\{x_{t}\right\}_{t=-\infty}^{\infty} \subset \Omega(a)$;

(2) for each $\varepsilon>0$ there exists a natural number $T(\varepsilon)$ such that for each trajectory $\left\{x_{t}\right\}_{t=0}^{\infty} \subset$ $X$ satisfying $\phi\left(x_{t}\right)=\phi\left(x_{t+1}\right)$ for all integers $t \geq 0$ the inequality $\rho\left(x_{t}, \Omega(a)\right) \leq \varepsilon$ holds for all integers $t \geq T(\varepsilon)$.

For each $x \in X$, set

$$
\pi(x)=\sup \left\{\lim _{t \rightarrow \infty} \phi\left(x_{t}\right):\left\{x_{t}\right\}_{t=0}^{\infty} \text { is a trajctory and } x_{0}=x\right\} .
$$

The function $\pi$ plays an important role in our study. The following two useful results were also obtained in [7].

Proposition 1.1. Let $x \in X$. Then there is a trajectory $\left\{x_{t}\right\}_{t=0}^{\infty}$ such that $x_{0}=x$, and $\pi(x)=$ $\lim _{t \rightarrow \infty} \phi\left(x_{t}\right)$.

Proposition 1.2. The function $\pi: X \rightarrow R^{1}$ is upper semicontinuous.

It is clear that, for each $x \in X$ and each $y \in a(x), \pi(y) \leq \pi(x)$, for each $x \in X \pi(x) \leq \phi(x)$, and that for each $x \in X$ and each natural number $n \pi(x) \leq \sup \left\{\phi(y): y \in a^{n}(x)\right\}$.

It is easy to see that the following proposition holds. 
Proposition 1.3. Let $x \in X$, and let $\left\{x_{t}\right\}_{t=0}^{\infty} \subset X$ be a trajectory such that $x_{0}=x$. Then $\lim _{t \rightarrow \infty} \phi\left(x_{t}\right)=\pi(x)$ if and only if, for each integer $t \geq 0, \pi\left(x_{t+1}\right)=\max \left\{\pi(z): z \in a\left(x_{t}\right)\right\}$.

The following useful result was also proved in [7].

Proposition 1.4. Let $x \in X$. Then $\pi(x)=\lim _{n \rightarrow \infty} \sup \left\{\phi(y): \in a^{n}(x)\right\}$.

The following theorem is the first turnpike result obtained in [7]. It describes the structure of optimal (with respect to the functional $\phi$ ) trajectories of $a$.

Theorem 1.2. Assume that the property (1) of Theorem 1.1 holds. Let $\varepsilon>0$ and $x \in X$. Then there exist $\delta>0$ and a natural number $L$ such that for each integer $T>2 L$ and each trajectory $\left\{x_{t}\right\}_{t=0}^{T}$ satisfying $x_{0}=x$ and $\phi\left(x_{T}\right) \geq \pi\left(x_{0}\right)-\delta$ the following inequality holds:

$$
\rho\left(x_{t}, \Omega(a)\right) \leq \varepsilon, t=L, \ldots, T-L .
$$

In this paper we use the following property introduced in [7].

(P) If $x_{1}, x_{2} \in \Omega(a)$, and $\phi\left(x_{1}\right)=\phi\left(x_{2}\right)$, then $x_{1}=x_{2}$.

Note that the property $(\mathrm{P})$ holds for many models of economic dynamics for which $\Omega(a)$ is a subinterval of a line $[1,8,9]$.

The next convergence result was established in [7].

Theorem 1.3. Assume that property $(P)$ holds. Then each trajectory of a converges to an element of $\Omega(a)$.

It is not difficult to see that the following result holds.

Proposition 1.5. Assume that property $(P)$ holds and that $\left\{x_{t}\right\}_{t=0}^{\infty}$ is a trajectory of a such that $\lim _{t \rightarrow \infty} \phi\left(x_{t}\right)=\pi(x)$. Then by Theorem 1.3, there exists $F(x)=\lim _{t \rightarrow \infty} x_{t}$, and the equality $\phi(F(x))=\lim _{t \rightarrow \infty} \phi\left(x_{t}\right)=\pi(x)$ holds. Moreover, $F(x)$ is a unique element of $\Omega(a)$ belonging to $\phi^{-1}(\pi(x))$.

In the sequel if property (P) holds, then, for each $x \in X$, we denote by $F(x)$ the unique element of $\Omega(a) \cap \phi^{-1}(\pi(x))$.

The following theorem is the second turnpike result obtained in [7]. It describes the structure of optimal (with respect to the functional $\phi$ ) trajectories of $a$.

Theorem 1.4. Assume that the property $(P)$ and property (1) of Theorem 1.1 hold. Let $\varepsilon>0$ and $x \in X$. Then there exist $\delta>0$ and a natural number $L$ such that for each integer $T>2 L$ and each trajectory $\left\{x_{t}\right\}_{t=0}^{T}$ satisfying $x_{0}=x$ and $\phi\left(x_{T}\right) \geq \pi(x)-\delta$, the following inequality holds:

$$
\rho\left(x_{t}, F(x)\right) \leq \varepsilon, t=L, \ldots, T-L .
$$

Theorems 1.2 and 1.4 establish the turnpike properties for approximate solutions of the problem

$$
\begin{gathered}
\phi\left(x_{T}\right) \rightarrow \max , \\
\left\{x_{t}\right\}_{t=0}^{T} \text { is a program satisfying } x_{0}=x,
\end{gathered}
$$

where $x \in X$ and a natural number $T$ are given. In Theorem 1.2, the turnpike is the set $\Omega(a)$ while in Theorem 1.4, the turnpike is a point $F(x)$.

In the present paper, our goal is to obtain generalizations of these results (Theorems 3.1 and 4.1) which show that the turnpike properties still hold in the case when $x_{0}$ is not necessarily $x$ 
but a point close to $x$. In Theorems 5.1 and 7.1, we establish strong versions of the turnpike when $x \in \Omega(a)$. In this case, for a program $\left\{x_{t}\right\}_{t=0}^{T}$ satisfying the assumptions of Theorem 1.2,

$$
\rho\left(x_{t}, \Omega(a)\right) \leq \varepsilon, t=0, \ldots, T-L .
$$

If it satisfies the assumptions of Theorem 1.4 , then $\rho\left(x_{t}, x\right) \leq \varepsilon, t=0, \ldots, T-L$. Note that in our results we assume that the function $\pi$ is continuous at $x$. As it follows from the results of [10], there exists an everywhere dense set $Y \subset X$ which is a countable intersection of open subsets of $X$ such that $\pi$ is continuous at all points which belong to $Y$.

It was also known that the function $\pi$ is continuous at $x \in X$ if the family of mappings $\left\{a^{n}\right.$ : $n=1,2, \ldots\}$ is equicontinuous at the point $x$ (see the next section). This result was obtained in [11].

It should be mentioned that turnpike properties are well known in mathematical economics. The term was first coined by Samuelson in 1948 (see [12]) where he showed that an efficient expanding economy would spend most of the time in the vicinity of a balanced equilibrium path (also called a von Neumann path and a turnpike). This property was further investigated for optimal trajectories of models of economic dynamics; see, e.g., [2, 8, 9] and the references mentioned there. Recently, it was shown that the turnpike phenomenon holds for many important classes of problems arising in various areas of research [13]-[19]. For related infinite horizon problems, we refer to [9, 20, 21, 22, 23, 24, 25].

The paper is organized as follows. Section 2 contains auxiliary results. Our first main result (Theorem 3.1) is proved in Section 3 while our second main results (Theorem 4.1) is established in Section 4. Our third main result (Theorem 5.1) is stated in Section 5. It follows from Proposition 5.1 which is also stated in Section 5 and is proved in Section 6. Our last main result (Theorem 7.1) is proved in Section 7. In Section 8, we present the main results of [11] which follow easily from Theorems 3.1, 4.1, 5.1, and 7.1.

\section{Auxiliary Results}

For each $x \in X$ and each $r>0$, set $B(x, r)=\{y \in X: \rho(x, y) \leq r\}$. It is clear that, for each integer $n \geq 1$, the graph of the mapping $a^{n}$ is a closed subset of $X \times X$. The collection of all nonempty subsets of $X$ is equipped with the Hausdorff distance defined for each pair $A, B \subset X$ by

$$
H(A, B)=\max \left\{\sup _{x \in A} \rho(x, B), \sup _{y \in B} \rho(y, A)\right\} .
$$

Let $x \in X$ and $n$ be a natural number. We say that the mapping $a^{n}$ is continuous at the point $x$ if, for each $\varepsilon>0$, there exists $\delta>0$ such that, for each point $y \in X$ satisfying $\rho(x, y) \leq \delta$,

$$
H\left(a^{n}(x), a^{n}(y)\right) \leq \varepsilon .
$$

Let $x \in X$. We say that the family of mappings $\left\{a^{n}: n=1,2, \ldots\right\}$ is equicontinuous at the point $x$ if, for each $\varepsilon>0$, there exists $\delta>0$ such that, for each natural number $n$ and each point $y \in X$ satisfying $\rho(x, y) \leq \delta, H\left(a^{n}(x), a^{n}(y)\right) \leq \varepsilon$.

In [11], we obtained the extensions of Theorems 1.2 and 1.4 under the assumption that the family of mappings $\left\{a^{n}: n=1,2, \ldots\right\}$ is equicontinuous at the point $x$. It should be mentioned that this assumption holds for some models of economic growth which are prototypes for discrete disperse dynamical systems. The following auxiliary result was obtained in [11]. 
Proposition 2.1. Assume that $x \in X$ and that the family of mappings $\left\{a^{n}: n=1,2, \ldots\right\}$ is equicontinuous at the point $x$. Then $\pi$ is continuous at $x$.

The next auxiliary result was also obtained in [11].

Proposition 2.2. Assume that the property (1) of Theorem 1.1 holds and that $z \in \Omega(a)$. Then there exists a sequence $\left\{y_{t}\right\}_{t=-\infty}^{\infty} \subset \Omega(a)$ such that $y_{0}=z, y_{t+1} \in a\left(y_{t}\right)$ for all integers $t$, and $\phi\left(y_{t}\right)=\phi\left(y_{0}\right)$ for all integers $t$.

Proposition 2.3. Assume that $x \in X$, the mapping $a^{n}(\cdot)$ is continuous at the point $x$ for all natural numbers $n$, the function $\pi$ is continuous at $x$, and that $\varepsilon>0$. Then there exist $\delta>0$ and a natural number $L$ such that, for each integer $T>L$ and each trajectory $\left\{x_{t}\right\}_{t=0}^{T}$ satisfying $\rho\left(x_{0}, x\right) \leq \delta$ and $\phi\left(x_{T}\right) \geq \pi\left(x_{0}\right)-\delta$, the following inequality holds:

$$
\left|\phi\left(x_{t}\right)-\pi(x)\right| \leq \varepsilon, t=L, \ldots, T .
$$

Proof. Since the function $\pi$ is continuous at $x$, there exists $\delta_{1} \in(0, \varepsilon / 4)$ such that

$$
|\pi(x)-\pi(y)| \leq \varepsilon / 8 \text { for all } y \in B\left(x, \delta_{1}\right) .
$$

Proposition 1.4 implies that there exists a natural number $L$ such that

$$
\left|\pi(x)-\sup \left\{\phi(y): y \in a^{L}(x)\right\}\right| \leq \varepsilon / 8 .
$$

By the uniform continuity of $\phi$, there exists $\delta_{2} \in\left(0, \delta_{1}\right)$ such that

$$
\begin{gathered}
\left|\phi\left(\xi_{1}\right)-\phi\left(\xi_{2}\right)\right| \leq \varepsilon / 8 \text { for all } \xi_{1}, \xi_{2} \in X \\
\text { satisfying } \rho\left(\xi_{1}, \xi_{2}\right) \leq \delta_{2} .
\end{gathered}
$$

By the continuity assumption, there exists $\delta \in\left(0, \delta_{2}\right)$ such that

$$
H\left(a^{L}(x), a^{L}(y)\right) \leq \delta_{2}
$$

for every $y \in B(x, \delta)$. Assume that $T>L$ is an integer and that a trajectory $\left\{x_{t}\right\}_{t=0}^{T}$ satisfies

$$
\rho\left(x_{0}, x\right) \leq \delta
$$

and

$$
\phi\left(x_{T}\right) \geq \pi\left(x_{0}\right)-\delta .
$$

By (1.1) and (2.6), for all integers $t=0, \ldots, T$, we have

$$
\phi\left(x_{t}\right) \geq \phi\left(x_{T}\right) \geq \pi\left(x_{0}\right)-\delta .
$$

In view of (2.1) and (2.5), we obtain

$$
\left|\pi(x)-\pi\left(x_{0}\right)\right| \leq \varepsilon / 8 .
$$

Equations (2.7) and (2.8) imply that, for all $t=0, \ldots, T$,

$$
\phi\left(x_{t}\right) \geq \pi\left(x_{0}\right)-\delta \geq \pi(x)-\delta-\varepsilon / 8 .
$$

It follows from (2.4) and (2.5) that

$$
z \in a^{L}(x)
$$

such that $\rho\left(z, x_{L}\right) \leq \delta_{2}$. By (2.3), we have

$$
\left|\phi(z)-\phi\left(x_{L}\right)\right| \leq \varepsilon / 8 .
$$


In view of (2.2), (2.10), and (2.11), we ahve

$$
\phi\left(x_{L}\right) \leq \phi(z)+\varepsilon / 8 \leq \sup \left\{\phi(\xi): \xi \in a^{L}(x)\right\}+\varepsilon / 8 \leq \pi(x)+\varepsilon / 4 .
$$

Equations (1.1) and (2.12) imply that, for all integers $t=L, \ldots, T, \phi\left(x_{t}\right) \leq \phi\left(x_{L}\right) \leq \pi(x)+\varepsilon / 4$, which together with (2.9) implies that $|\phi(x)-\pi(x)| \leq \varepsilon$ for all integers $t=L, \ldots, T$. Proposition 2.3 is proved.

\section{The First Main Result}

Theorem 3.1. Assume that the property (1) of Theorem 1.1 holds, $x \in X$, the mapping $a^{n}$ is continuous at the point $x$ for all natural numbers $n$, the function $\pi$ is continuous at $x$, and $\varepsilon>0$. Then there exist $\delta>0$, and a natural number $L$ such that, for each integer $T>2 L$ and each trajectory $\left\{x_{t}\right\}_{t=0}^{T}$ satisfying $\rho\left(x_{0}, x\right) \leq \delta$ and $\phi\left(x_{T}\right) \geq \pi\left(x_{0}\right)-\delta$, the following inequalities hold:

$$
\left|\phi\left(x_{t}\right)-\pi(x)\right| \leq \varepsilon, t=L, \ldots, T,
$$

and

$$
\rho\left(x_{t}, \Omega(a)\right) \leq \varepsilon, t=L, \ldots, T-L .
$$

Theorem 3.1 easily follows from Proposition 2.3 and the following result.

Theorem 3.2. Assume that the property (1) of Theorem 1.1 holds, $x \in X$, the mapping $a^{n}$ is continuous at the point $x$ for all natural numbers $n$, the function $\pi$ is continuous at $x$, and $\varepsilon>0$. Then there exist $\delta>0$ and a natural number $L$ such that, for each integer $T>2 L$ and each trajectory $\left\{x_{t}\right\}_{t=0}^{T}$ satisfying $\rho\left(x_{0}, x\right) \leq \delta$ and $\phi\left(x_{T}\right) \geq \pi\left(x_{0}\right)-\delta$, the following inequality holds:

$$
\rho\left(x_{t}, \Omega(a)\right) \leq \varepsilon, t=L, \ldots, T-L .
$$

Proof. By Proposition 2.3, for every natural number $n$, there exist $\delta_{n} \in\left(0, n^{-1}\right)$ and a natural number $L_{n}$ such that the following property holds:

(a) for each integer $T>L_{n}$ and each trajectory $\left\{x_{t}\right\}_{t=0}^{T}$ satisfying $\rho\left(x_{0}, x\right) \leq \delta_{n}$, and $\phi\left(x_{T}\right) \geq$ $\pi\left(x_{0}\right)-\delta_{n}$, we have $\left|\phi\left(x_{t}\right)-\pi(x)\right| \leq 1 / n, t=L_{n}, \ldots, T$. Assume that the theorem does not hold. Then for each natural number $n$ there exist an integer

$$
T_{n}>4 n+4 L_{n}+4
$$

and a trajectory $\left\{x_{t}^{(n)}\right\}_{t=0}^{T_{n}}$ satisfying

$$
\begin{gathered}
\rho\left(x_{0}^{(n)}, x\right) \leq \delta_{n}, \\
\phi\left(x_{T_{n}}^{(n)}\right) \geq \pi\left(x_{0}^{(n)}\right)-\delta_{n},
\end{gathered}
$$

and

$$
\max \left\{\rho\left(x_{t}^{(n)}, \Omega(a)\right): t=2 n+2 L_{n}, \ldots, T_{n}-2 n-2 L_{n}\right\}>\varepsilon .
$$

For each natural number $n$, it follow from (3.4) that there exist an integer

$$
S_{n} \in\left[2 n+2 L_{n}, T_{n}-2 n-2 L_{n}\right]
$$

such that $\rho\left(x_{S_{n}}^{(n)}, \Omega(a)\right)>\varepsilon$. Let $n \geq 1$ be an integer. Property (a) and (3.1)-(3.3) imply that

$$
\left|\phi\left(x_{t}^{(n)}\right)-\pi(x)\right| \leq 1 / n, t=L_{n}, \ldots, T_{n} .
$$


Set

which implies that

$$
y_{t}^{(n)}=x_{t+S_{n}}^{(n)}, t=-S_{n}, \ldots, T_{n}-S_{n}
$$

$$
\rho\left(y_{0}^{(n)}, \Omega(a)\right)=\rho\left(x_{S_{n}}^{(n)}, \Omega(a)\right)>\varepsilon .
$$

It follows from (3.5) and (3.6) that, for all integers $t=L_{n}-S_{n}, \ldots, T_{n}-S_{n}$,

$$
\left|\phi\left(y_{t}^{(n)}\right)-\pi(x)\right|=\left|\phi\left(x_{t+S_{n}}^{(n)}\right)-\pi(x)\right| \leq 1 / n
$$

Extracting subsequences and using diagonalization process, we obtain that there exists a strictly increasing sequence of natural numbers $\left\{n_{j}\right\}_{j=1}^{\infty}$ such that, for each integer $t$, there exists $y_{t}=$ $\lim _{j \rightarrow \infty} y_{t}^{\left(n_{j}\right)}$. Since the graph of $a$ is closed, we have that

$$
y_{t+1} \in a\left(y_{t}\right) \text { for all integers } t \text {. }
$$

By (3.7), we have

$$
\rho\left(y_{0}, \Omega(a)\right) \geq \varepsilon .
$$

From (3.8), we have that, for all integers $t, \phi\left(y_{t}\right)=\pi(x)$. From (3.9) and the property (1) of Theorem 1.1, this implies that $y_{t} \in \Omega(a)$ for all integers $t$. This contradicts (3.10). The contradiction we have reached proves Theorem 3.2.

\section{The Second Main Result}

Theorem 4.1. Assume that the property $(P)$ and property (1) of Theorem 1.1 hold, $x \in X$, the mapping $a^{n}$ is continuous at $x$ for all natural numbers $n$, the function $\pi$ is is continuous at $x$, and $t \varepsilon>0$. Then there exist $\delta>0$ and a natural number $L$ such that, for each integer $T>2 L$ and each trajectory $\left\{x_{t}\right\}_{t=0}^{T}$ satisfying $\rho\left(x_{0}, x\right) \leq \delta$ and $\phi\left(x_{T}\right) \geq \pi\left(x_{0}\right)-\delta$, the following inequalities hold:

$$
\begin{gathered}
\rho\left(x_{t}, F(x)\right) \leq \varepsilon, t=L, \ldots, T-L \\
\left|\phi\left(x_{t}\right)-\pi(x)\right| \leq \varepsilon, t=L, \ldots, T .
\end{gathered}
$$

Proof. From property $(\mathrm{P})$, we see that there is $\varepsilon_{0} \in(0, \varepsilon / 4)$ such that the following property holds:

(P1)

$$
\text { if } z_{1}, z_{2} \in \Omega(a) \text { satisfy }\left|\phi\left(z_{1}\right)-\phi\left(z_{2}\right)\right| \leq 4 \varepsilon_{0} \text {, then } \rho\left(z_{1}, z_{2}\right) \leq \varepsilon / 4 .
$$

Since $\phi$ is uniformly continuous on $X$ there is $\varepsilon_{1} \in\left(0, \varepsilon_{0} / 4\right)$ such that

$$
\left|\phi\left(z_{1}\right)-\phi\left(z_{2}\right)\right| \leq \varepsilon_{0} / 4
$$

for each $z_{1}, z_{2} \in X$ satisfying $\rho\left(z_{1}, z_{2}\right) \leq \varepsilon_{1}$, From Theorem 3.1, we see that there exist $\delta \in$ $\left(0, \varepsilon_{1} / 2\right)$, and a natural number $L$ such that the following property holds:

(P2) For each integer $T>2 L$ and each trajectory $\left\{x_{t}\right\}_{t=0}^{T}$ satisfying

$$
\begin{gathered}
\rho\left(x_{0}, x\right) \leq \delta, \\
\phi\left(x_{T}\right) \geq \pi\left(x_{0}\right)-\delta,
\end{gathered}
$$

we have

$$
\rho\left(x_{t}, \Omega(a)\right) \leq \varepsilon_{1}, t=L, \ldots, T-L,
$$

and

$$
\left|\phi\left(x_{t}\right)-\pi(x)\right| \leq \varepsilon_{1}, t=L, \ldots, T
$$


Assume that an integer $T>2 L$, and a trajectory $\left\{x_{t}\right\}_{t=0}^{T}$ satisfies (4.2) and (4.3). By property (P2), equations (4.4) and (4.5) hold. In view of (4.5) and the choice of $\varepsilon_{0}, \varepsilon_{1}$, we have

$$
\left|\phi\left(x_{t}\right)-\pi(x)\right| \leq \varepsilon, t=L, \ldots, T .
$$

Assume that an integer $t \in[L, T-L]$. It follows from (4.4) that there is $z \in \Omega(a)$ such that

$$
\rho\left(x_{t}, z\right) \leq \varepsilon_{1} .
$$

In view of (4.5), we have

$$
\left|\phi\left(x_{t}\right)-\pi(x)\right| \leq \varepsilon_{1}
$$

From (4.1) and (4.6), we have

$$
\left|\phi(z)-\phi\left(x_{t}\right)\right| \leq \varepsilon_{0} / 4
$$

Hence,

$$
F(x) \in \Omega(a), \phi(F(x))=\pi(x) .
$$

It follows from (4.7)-(4.9) that

$$
\begin{aligned}
|\phi(z)-\phi(F(x))| & =|\phi(z)-\pi(x)| \\
& \leq\left|\phi(z)-\phi\left(x_{t}\right)\right|+\left|\phi\left(x_{t}\right)-\pi(x)\right| \\
& \leq \varepsilon_{0} / 4+\varepsilon_{1} \leq \varepsilon_{0} .
\end{aligned}
$$

Property (P1) and (4.9) imply that $\rho(z, F(x)) \leq \varepsilon / 4$, which together with (4.6) yields that

$$
\rho\left(x_{t}, F(x)\right) \leq \rho\left(x_{t}, z\right)+\rho(z, F(x)) \leq \varepsilon .
$$

This completes the proof.

\section{The ThiRd MAin Result}

Theorem 5.1. Assume that the property (1) of Theorem 1.1 holds, $x \in \Omega(a)$, the mapping $a^{n}$ is continuous at the point $x$ for all natural numbers $n$, the function $\pi$ is continuous at $x$, and $\varepsilon>0$. Then there exist $\delta>0$ and a natural number $L$ such that, for each integer $T>2 L$ and each trajectory $\left\{x_{t}\right\}_{t=0}^{T}$ satisfying $\rho\left(x_{0}, x\right) \leq \delta$ and $\phi\left(x_{T}\right) \geq \pi\left(x_{0}\right)-\delta$, the following inequalities hold:

$$
\left|\phi\left(x_{t}\right)-\pi(x)\right| \leq \varepsilon, t=0, \ldots, T
$$

and

$$
\rho\left(x_{t}, \Omega(a)\right) \leq \varepsilon, t=0, \ldots, T-L .
$$

Theorem 5.1 follows from Theorem 3.1 and the following proposition which is proved in the next section.

Proposition 5.1. Assume that the property (1) of Theorem 1.1 holds, $x \in \Omega(a)$, the mapping $a^{n}$ is continuous at the point $x$ for all natural numbers $n$, the function $\pi$ is continuous at $x, L_{0}$ is a natural number, and $\varepsilon>0$. Then there exist $\delta>0$ and a natural number $L>L_{0}$ such that, for each integer $T>L$ and each trajectory $\left\{x_{t}\right\}_{t=0}^{T}$ satisfying $\rho\left(x_{0}, x\right) \leq \delta$ and $\phi\left(x_{T}\right) \geq \pi\left(x_{0}\right)-\delta$, the following inequalities hold:

$$
\left|\phi\left(x_{t}\right)-\pi(x)\right| \leq \varepsilon, t=0, \ldots, L_{0}
$$

and

$$
\rho\left(x_{t}, \Omega(a)\right) \leq \varepsilon, t=0, \ldots, L_{0}
$$




\section{ProOF OF Proposition 5.1}

In the proof of Proposition 5.1, we use the following auxiliary result.

Lemma 6.1. Let $\varepsilon>0$. Then there exist $\delta>0$ such that, for each integer $T$ and each trajectory $\left\{x_{t}\right\}_{t=0}^{T}$ satisfying $\rho\left(x_{0}, x\right) \leq \delta$ and $\phi\left(x_{T}\right) \geq \pi\left(x_{0}\right)-\delta$, the following inequalities hold:

$$
\left|\phi\left(x_{t}\right)-\pi(x)\right| \leq \varepsilon, t=0, \ldots, T .
$$

Proof. Since the function $\pi$ is continuous at $x$ there is $\delta_{1} \in(0, \varepsilon / 4)$ such that

$$
|\pi(x)-\pi(y)| \leq \varepsilon / 8 \text { for all } y \in B\left(x, \delta_{1}\right) .
$$

Proposition 2.2 and (1.2) imply that $\pi(x)=\phi(x)$. Since $\phi$ is uniformly continuous on $X$, there is $\delta \in\left(0, \delta_{1}\right)$ such that

$$
\left|\phi\left(\xi_{1}\right)-\phi\left(\xi_{2}\right)\right| \leq \varepsilon / 8
$$

for each $\xi_{1}, \xi_{2} \in X$ satisfying $\rho\left(\xi_{1}, \xi_{2}\right) \leq \delta$. Assume that $T$ is a natural number and that a trajectory $\left\{x_{t}\right\}_{t=0}^{T}$ satisfies

$$
\rho\left(x_{0}, x\right) \leq \delta
$$

and

$$
\phi\left(x_{T}\right) \geq \pi\left(x_{0}\right)-\delta .
$$

It follows from (1.1) and (6.4) that, for all integers $t=0, \ldots, T$,

$$
\phi\left(x_{t}\right) \geq \phi\left(x_{T}\right) \geq \pi\left(x_{0}\right)-\delta .
$$

By (6.1) and (6.3), we have

$$
\left|\pi(x)-\pi\left(x_{0}\right)\right| \leq \varepsilon / 8 .
$$

In view of (6.5) and (6.6), for all integers $t=0, \ldots, T$, we have

$$
\phi\left(x_{t}\right) \geq \pi\left(x_{0}\right)-\delta \geq \pi(x)-\delta-\varepsilon / 8 .
$$

Equations (6.2) and (6.3) imply that

$$
\left|\phi\left(x_{0}\right)-\phi(x)\right| \leq \varepsilon / 8 .
$$

It follows from (1.1) and (6.7) that, for all integers $t=0, \ldots, T$,

$$
\phi\left(x_{t}\right) \leq \phi\left(x_{0}\right) \leq \phi(x)+\varepsilon / 8=\pi(x)+\varepsilon / 8,
$$

which together with (6.5) implies that, for all $t=0, \ldots, T,\left|\phi\left(x_{t}\right)-\pi(x)\right| \leq \varepsilon$. This completes the proof.

Proposition 5.1 easily follows from Lemma 6.1 and the following result.

Proposition 6.1. Assume that the property (1) of Theorem 1.1 holds, $x \in \Omega(a)$, the mapping $a^{n}$ is continuous at the point $x$ for all natural numbers $n$, the function $\pi$ is continuous at $x, L_{0}$ is a natural number, and $\varepsilon>0$. Then there exist $\delta>0$ and a natural number $L>L_{0}$ such that, for each integer $T>L$ and each trajectory $\left\{x_{t}\right\}_{t=0}^{T}$ satisfying $\rho\left(x_{0}, x\right) \leq \delta$ and $\phi\left(x_{T}\right) \geq \pi\left(x_{0}\right)-\delta$, the following inequalities hold:

$$
\rho\left(x_{t}, \Omega(a)\right) \leq \varepsilon, t=0, \ldots, L_{0} .
$$


Proof. By Lemma 6.1, for each natural number $n$ there exists $\delta_{n} \in\left(0, n^{-1}\right)$ such that the following property holds:

(a) for each integer $T$ and each trajectory $\left\{x_{t}\right\}_{t=0}^{T}$ satisfying $\rho\left(x_{0}, x\right) \leq \delta_{n}$ and $\phi\left(x_{T}\right) \geq$ $\pi\left(x_{0}\right)-\delta_{n}$, the following inequalities hold:

$$
\left|\phi\left(x_{t}\right)-\pi(x)\right| \leq 1 / n, t=0, \ldots, T .
$$

Assume that the proposition does not hold. Then, for each natural number $n$, there exist an integer $T_{n} \geq 4\left(n+L_{0}+1\right)$ and a trajectory $\left\{x_{t}^{(n)}\right\}_{t=0}^{T_{n}}$ satisfying

$$
\begin{gathered}
\rho\left(x_{0}^{(n)}, x\right) \leq \delta_{n}, \\
\phi\left(x_{T_{n}}^{(n)}\right) \geq \pi\left(x_{0}^{(n)}\right)-\delta_{n},
\end{gathered}
$$

and

$$
\max \left\{\rho\left(x_{t}^{(n)}, \Omega(a)\right): t=0, \ldots, L_{0}\right\}>\varepsilon .
$$

For each natural number $n$, it follows from (6.10) there exist an integer $S_{n} \in\left[0, L_{0}\right]$ such that

$$
\rho\left(x_{S_{n}}^{(n)}, \Omega(a)\right)>\varepsilon .
$$

Let $n$ be natural number. Property (a), (6.8) and (6.9) imply that

$$
\left|\phi\left(x_{t}^{(n)}\right)-\pi(x)\right| \leq n^{-1}, t=0, \ldots, T_{n} .
$$

Extracting subsequences and using diagonalization process, we obtain that there exists a strictly increasing sequence of natural numbers $\left\{n_{j}\right\}_{j=1}^{\infty}$ such that for each integer $t$ there exists

$$
x_{t}=\lim _{j \rightarrow \infty} x_{t}^{\left(n_{j}\right)}, S_{n_{j}}=S_{n_{1}} \text { for all natural numbers } j .
$$

Since the graph of the mapping $a$ is closed, for every integer $t \geq 0$, we have $x_{t+1} \in a\left(x_{t}\right)$. By (6.11)-(6.13), we have

$$
\rho\left(x_{S_{n_{1}}}, \Omega(a)\right) \geq \varepsilon
$$

In view of (6.11)-(6.13), we obtain $\phi\left(x_{t}\right)=\pi(x)$ for all integers $t \geq 0$. By Proposition 2.2, for every integer $t<0$, we have that there is $x_{t} \in X$ such that, for all integers $t, x_{t+1} \in a\left(x_{t}\right)$, and $\phi\left(x_{t}\right)=\phi\left(x_{0}\right)$. From the property (1) of Theorem 1.1, this implies that $x_{t} \in \Omega(a)$ for all integers $t$. This contradicts (6.14). The contradiction we have reached completes the proof of this proposition

\section{The Fourth MAIN Result}

Theorem 7.1. Assume that property $(P)$ and the property (1) of Theorem 1.1 hold, $x \in \Omega(a)$, the mapping $a^{n}$ is continuous at the point $x$ for all natural numbers $n$, the function $\pi$ is continuous at $x$, and and $\varepsilon>0$. Then there exist $\delta>0$ and a natural number $L$ such that, for each integer $T>2 L$ and each trajectory $\left\{x_{t}\right\}_{t=0}^{T}$ satisfying $\rho\left(x_{0}, x\right) \leq \delta$ and $\phi\left(x_{T}\right) \geq \pi\left(x_{0}\right)-\delta$, the following inequalities hold:

$$
\rho\left(x_{t}, x\right) \leq \varepsilon, t=0, \ldots, T-L
$$

and

$$
\left|\phi\left(x_{t}\right)-\pi(x)\right| \leq \varepsilon, t=0, \ldots, T .
$$


Proof. By the property $(\mathrm{P})$, there is $\varepsilon_{0} \in(0, \varepsilon / 4)$ such that the following property holds:

(P1)

$$
\text { if } z_{1}, z_{2} \in \Omega(a) \text { satisfy }\left|\phi\left(z_{1}\right)-\phi\left(z_{2}\right)\right| \leq \varepsilon_{0}, \text { then } \rho\left(z_{1}, z_{2}\right) \leq \varepsilon / 4 .
$$

Since $\phi$ is uniformly continuous on $X$, there is $\varepsilon_{1} \in\left(0, \varepsilon_{0} / 4\right)$ such that

$$
\left|\phi\left(z_{1}\right)-\phi\left(z_{2}\right)\right| \leq \varepsilon_{0} / 4
$$

for all $z_{1}, z_{2} \in X$ satisfying $\rho\left(z_{1}, z_{2}\right) \leq \varepsilon_{1}$.

Theorem 5.1 implies that there exist a natural number $L$ and a positive number $\delta<\varepsilon_{1} / 2$ such that the following property holds:

(P2) for each integer $T>2 L$ and each trajectory $\left\{x_{t}\right\}_{t=0}^{T}$ satisfying

$$
\rho\left(x_{0}, x\right) \leq \delta
$$

and

$$
\phi\left(x_{T}\right) \geq \pi\left(x_{0}\right)-\delta
$$

we have

$$
\rho\left(x_{t}, \Omega(a)\right) \leq \varepsilon_{1}, t=0, \ldots, T-L,
$$

and

$$
\left|\phi\left(x_{t}\right)-\pi(x)\right| \leq \varepsilon_{1}, t=0, \ldots, T .
$$

Assume that an integer $T>2 L$ and that a trajectory $\left\{x_{t}\right\}_{t=0}^{T}$ satisfies (7.2) and (7.3). In view of (P2), we have (7.4) and (7.5) hold. Proposition 2.2 implies that

$$
\pi(x)=\phi(x) .
$$

We have

$$
x \in \Omega(a) .
$$

Propositions 1.5 and 2.2, (7.6), and (7.7) imply that $x=F(x)$. Assume $t \in[0, T-L]$. It follows from (7.4) that there is $z \in \Omega(a)$ such that $\rho\left(x_{t}, z\right) \leq \varepsilon_{1}$. In view of (7.1) and (7.5), we have $\left|\phi\left(x_{t}\right)-\pi(x)\right| \leq \varepsilon_{1}$ and $\left|\phi(z)-\phi\left(x_{t}\right)\right| \leq \varepsilon_{0} / 4$, which together with (7.6) imply that

$$
|\phi(z)-\phi(x)| \leq \varepsilon_{0} / 4+\left|\phi\left(x_{t}\right)-\pi(x)\right| \leq \varepsilon_{0} / 4+\varepsilon_{1} \leq \varepsilon_{0} / 2 .
$$

From Property (P1) and (7.7), we have $\rho(z, x)<\varepsilon / 4$. Hence, $\rho\left(x_{t}, x\right) \leq \varepsilon$. This completes the proof.

\section{Applications}

It is easy to see that Theorems 3.1, 4.1, 5.1, and 7.1, respectively, combining with Proposition 2.1, implies the following main results of [11].

Theorem 8.1. Assume that the property (1) of Theorem 1.1 holds, $x \in X$, the family of mappings $\left\{a^{n}: n=1,2, \ldots\right\}$ is equicontinuous at the point $x$, and $t \varepsilon>0$. Then there exist $\delta>0$ and a natural number $L$ such that, for each integer $T>2 L$ and each trajectory $\left\{x_{t}\right\}_{t=0}^{T}$ satisfying $\rho\left(x_{0}, x\right) \leq \delta$ and $\phi\left(x_{T}\right) \geq \pi\left(x_{0}\right)-\delta$, the following inequality holds:

$$
\begin{gathered}
\rho\left(x_{t}, \Omega(a)\right) \leq \varepsilon, t=L, \ldots, T-L \\
\left|\phi\left(x_{t}\right)-\pi(x)\right| \leq \varepsilon, t=L, \ldots, T .
\end{gathered}
$$


Theorem 8.2. Assume that the property $(P)$ and the property (1) of Theorem 1.1 hold, $x \in X$, the family of mappings $\left\{a^{n}: n=1,2, \ldots\right\}$ is equicontinuous at the point $x$, and $\varepsilon>0$. Then there exist $\delta>0$ and a natural number $L$ such that, for each integer $T>2 L$ and each trajectory $\left\{x_{t}\right\}_{t=0}^{T}$ satisfying $\rho\left(x_{0}, x\right) \leq \delta$ and $\phi\left(x_{T}\right) \geq \pi\left(x_{0}\right)-\delta$, the following inequalities hold:

$$
\begin{gathered}
\rho\left(x_{t}, F(x)\right) \leq \varepsilon, t=L, \ldots, T-L, \\
\left|\phi\left(x_{t}\right)-\pi(x)\right| \leq \varepsilon, t=L, \ldots, T .
\end{gathered}
$$

Theorem 8.3. Assume that the property (1) of Theorem 1.1 holds, $x \in \Omega(a)$, the family of mappings $\left\{a^{n}: n=1,2, \ldots\right\}$ is equicontinuous at the point $x$, and $\varepsilon>0$. Then there exist $\delta>0$ and a natural number $L$ such that, for each integer $T>L$ and each trajectory $\left\{x_{t}\right\}_{t=0}^{T}$ satisfying $\rho\left(x_{0}, x\right) \leq \delta$ and $\phi\left(x_{T}\right) \geq \pi\left(x_{0}\right)-\delta$, the following inequalities hold:

$$
\begin{gathered}
\rho\left(x_{t}, \Omega(a)\right) \leq \varepsilon, t=0, \ldots, T-L \\
\left|\phi\left(x_{t}\right)-\pi(x)\right| \leq \varepsilon, t=0, \ldots, T .
\end{gathered}
$$

Theorem 8.4. Assume that property $(P)$ and the property (1) of Theorem 1.1 hold, $x \in \Omega(a)$, the family of mappings $\left\{a^{n}: n=1,2, \ldots\right\}$ is equicontinuous at the point $x$, and $\varepsilon>0$. Then there exist $\delta>0$ and a natural number $L$ such that, for each integer $T>L$ and each trajectory $\left\{x_{t}\right\}_{t=0}^{T}$ satisfying $\rho\left(x_{0}, x\right) \leq \delta$ and $\phi\left(x_{T}\right) \geq \pi\left(x_{0}\right)-\delta$, the following inequalities hold:

$$
\begin{gathered}
\rho\left(x_{t}, F(x)\right) \leq \varepsilon, t=0, \ldots, T-L \\
\left|\phi\left(x_{t}\right)-\pi(x)\right| \leq \varepsilon, t=0, \ldots, T .
\end{gathered}
$$

\section{REFERENCES}

[1] A. M. Rubinov, Turnpike sets in discrete disperse dynamical systems, Sib. Math. J. 21 (1980), 136-146.

[2] A. M. Rubinov, Multivalued Mappings and Their Applications in Economic Mathematical Problems, Nauka, Leningrad, 1980.

[3] Z. Dzalilov, A. J. Zaslavski, Global attractors for discrete disperse dynamical systems, J. Nonlinear Convex Anal. 10 (2009), 191-198.

[4] A. J. Zaslavski, Turnpike sets of continuous transformations in compact metric spaces, Sib. Math. J. 23 (1982), 136-146.

[5] A. J. Zaslavski, Uniform convergence to global attractors for discrete disperse dynamical systems, Nonlinear Dyn. Sys. Theory 4 (2007), 315-325.

[6] A. J. Zaslavski, Convergence of trajectories of discrete dispersive dynamical systems, Commun. Mathe. Anal. 4 (2008), 10-19.

[7] A. J. Zaslavski, Structure of trajectories of discrete dispersive dynamical systems, Commun. Math. Anal. 6 2009, 1-9.

[8] V. L. Makarov, A. M. Rubinov, Mathematical Theory of Economic Dynamics and Equilibria, Springer-Verlag, New York, 1977.

[9] A. J. Zaslavski, Turnpike Properties in the Calculus of Variations and Optimal Control, Springer Optimization and Its Applications, New York, 2006.

[10] A. J. Zaslavski, Generic continuity of a lower semicontinuous function, PanAmer. Math. J. 16 (2006), 97-106.

[11] A. J. Zaslavski, Turnpike properties of discrete dispersive dynamical systems with a Lyapunov function, Optimization, in press.

[12] P. A. Samuelson, A catenary turnpike theorem involving consumption and the golden rule, Amer. Econom. Rev. 55 (1965), 486-496.

[13] T. Damm, L. Grune, M. Stieler, K. Worthmann, An exponential turnpike theorem for dissipative discrete time optimal control problems, SIAM J. Control Optim. 52 (2014), 1935-1957. 
[14] M. Gugat, E. Trelat, E. Zuazua, Optimal Neumann control for the 1D wave equation: finite horizon, infinite horizon, boundary tracking terms and the turnpike property, Systems Control Lett. 90 (2016), 61-70.

[15] M. Ali Khan, A. J. Zaslavski, On two classical turnpike results for the Robinson-Solow-Srinivisan (RSS) model, Adv. Math. Econom. 13 (2010), 47-97.

[16] M. Mammadov, Turnpike theorem for an infinite horizon optimal control problem with time delay, SIAM J. Control Optim. 52 (2014), 420-438.

[17] A. Porretta, E. Zuazua, Long time versus steady state optimal control, SIAM J. Control Optim. 51 (2013), $4242-4273$.

[18] E. Trelat, E. Zuazua, The turnpike property in finite-dimensional nonlinear optimal control, J. Differential Equations 218 (2015), 81-114.

[19] A. J. Zaslavski, Turnpike Theory of Continuous-time Linear Optimal Control Problems, Springer Optimization and Its Applications, Springer, Cham-Heidelberg-New York-Dordrecht-London, 2015.

[20] M. Bachir, J. Blot, Infinite dimensional infinite-horizon Pontryagin principles for discrete-time problems, Set-Valued Var. Anal. 23 (2015), 43-54.

[21] M. Bachir, J. Blot, Infinite dimensional multipliers and Pontryagin principles for discrete-time problems, Pure Appl. Funct. Anal. 2 (2017) , 411-426.

[22] J. Blot, N. Hayek, Infinite-horizon Optimal Control in the Discrete-time Framework, SpringerBriefs in Optimization, New York, 2014.

[23] D. A. Carlson, A. Haurie, A. Leizarowitz, Infinite Horizon Optimal Control, Berlin, Springer-Verlag, 1991.

[24] V. Gaitsgory, L. Grune, N. Thatcher, Stabilization with discounted optimal control, Systems Control Lett. 82 (2015), 91-98.

[25] N. Sagara, Recursive variational problems in nonreflexive Banach spaces with an infinite horizon: an existence result, Discrete Contin. Dyn. Syst. Ser. S 11 (2018), 1219-1232. 